\title{
PANDEMIA DA COVID-19: PESQUISA DOCUMENTAL A PARTIR DE PUBLICAÇÕES DO CONSELHO FEDERAL DE ENFERMAGEM
}

Samira Silva Santos Soares ${ }^{1}$

Norma Valéria Dantas de Oliveira Souza ${ }^{2}$

Karla Gualberto Silva ${ }^{1}$

Carolina Cabral Pereira da Costa ${ }^{2}$

Pacita Geovana Gama de Sousa Aperibense ${ }^{3}$

Ana Paula da Costa Lacerda Brandão ${ }^{1}$

Ana Cristina Silva de Carvalho ${ }^{1}$

Alessandra Cabral de Lacerda https://orcid.org/ 0000-0001-9133-7044 https://orcid.org/0000-0002-2936-3468 https://orcid.org/ 0000-0002-7870-0600 https://orcid.org/0000-0002-0365-7580 https://orcid.org/ 0000-0002-3176-2134 https://orcid.org/0000-0002-6255-3714 https://orcid.org/0000-0002-4303-4740 https://orcid.org/0000-0002-1408-7835

Objetivo: analisar o conteúdo relacionado à COVID-19 veiculado nas publicações do Conselho Federal de Enfermagem (Cofen). Método: Pesquisa documental cujas fontes primárias foram reportagens enotícias divulgadas no sítio eletrônico do Conselho Federal de Enfermagem. O recorte temporal compreende o periodo de dois meses. A partir da análise dos dados, definiu-se categorias temáticas que permitiram uma análise pormenorizada dos registros. Resultados: Foram analisadas 113 publicações, cujos conteúdos versavam sobre o avanço da pandemia no país, divulgava materiais de cunho informativo, educativos e científico sobre a doença, registravam a luta em prol da proteção dos serviços de saúde, segurança e saúde detrabalhadores e da sociedade, além de apontar para os desafios impostos pela crise em curso eas preocupaçõesadvindasdetalsituaçãoparaaatuaçãodoprofissionaldaenfermagem. Conclusões: Omaterialpublicadoauxiliaosprofissionais da enfermagem a compreenderem a pandemia e suas implicações para o trabalho da enfermagem, instrumentaliza-os com materiais de qualidade, garantindo-lhes um respaldo para a prática, além de deixar em evidência as ações e decisões do Cofen durante a pandemia. Descritores: Pandemias; Coronavirus; Enfermagem; Notícias; Exercício Profissional.

\section{COVID-19 PANDEMIC: DOCUMENTARY RESEARCH FROM PUBLICATIONS OF THE FEDERAL NURSING COUNCIL}

Objective: analyze the content related to COVID-19 published in the publications of the Federal Nursing Council. Method: Documentary research whose primary sources were reports and news published on the website of the Federal Nursing Council. The time frame comprises the period of two months. From the data analysis, thematic categories were defined that allowed a detailed analysis of the records. Results: A total of 113 publications were analyzed, whose contents focused on the advance of the pandemic in the country, disseminated informative, educational and scientific materials about the disease, recorded the struggle for the protection of health services, safety and health of workers and society, in addition to pointing to the challenges imposed by the current crisis and the concerns arising from this situation for the performance of the nursing professional. Conclusions: The published material helps nursing professionals to understand the pandemic and its implications for nursing work and equips them with quality materials and ensuring them a support for practice, in addition to instrumentalizing the professional collective to claim better working conditions. Descriptors: Pandemics; Coronavirus; Nursing; News; Professional Practice.

\section{COVID-19 PANDEMIC: INVESTIGACIÓN DOCUMENTAL DE PUBLICACIONES DEL CONSEJO FEDERAL DE ENFERMERÍA}

Objetivo: analizar el contenido relacionado con COVID-19 publicado en las publicaciones del Consejo Federal de Enfermería. Metodo: Investigación documental cuyas fuentes principales fueron informes y noticias publicadas en el sitio web del Consejo Federal de Enfermería. El plazo comprende el período de dos meses. A partir del análisis de datos, se definieron categorías temáticas que permitieron un análisis detallado de los registros. Resultados: Fueron analizados 113 publicaciones, cuyos contenidos se centraron en el avance de la pandemia en el país, difundimos materiales informativos, educativos y científicos sobre la enfermedad, registramos la lucha por la protección de los servicios de salud, la seguridad y la salud de los trabajadores y la sociedad, además de señalar los retos impuestos por la crisis actual y las preocupaciones derivadas de estasituación para el desempeño delprofesionaldeenfermería. Conclusiones: Elmaterialpublicado ayudaa los profesionales de enfermería a comprender la pandemia y sus implicaciones para el trabajo de enfermería y les dota de materiales de calidady les garantiza un apoyo a la práctica, además de instrumentalizar al colectivo profesional para que reprenda mejores condiciones de trabajo. Descriptores: Pandemias; Coronavirus; Enfermería; Noticias; Práctica Profesional.

${ }^{1}$ Universidade Federal do Rio de Janeiro, Escola de Enfermagem Anna Nery, Rio de Janeiro, RJ

2 Universidade do Estado do Rio de Janeiro, Faculdade de Enfermagem, Rio de Janeiro, RJ.

${ }^{3}$ Universidade do Estado do Rio de Janeiro, campus Professor Aloísio Teixeira, Macaé/RJ - Brasil

Autor Correspondente: Samira Silva Santos Soares. E-mail: samira_opg@hotmail.com

Recebido: 29/4/2020 - Aceito: 28/5/2020 


\section{INTRODUÇÃO}

No final de janeiro de 2020, a Organização Mundial de Saúde (OMS) declarou o surto da doença identificada como COVID-19, causada pelo novo coronavírus (SARS-Cov-2), caracterizando-a como uma emergência de saúde pública de importância internacional(1-2). Em 11 de março, à COVID-19 foi classificada pela OMS como uma pandemia(1). E, desde então, a imprensa mundial passou a disseminar massivamente notícias a respeito da doença, cumprindo seu papel de alertar o público sobre a possibilidade de um colapso do sistema de saúde e de uma crise sanitária severa, que poderia gerar uma elevada morbimortalidade de milhões de indivíduos ${ }^{(3-4)}$.

Todavia, no Brasil, apesar do avanço da doença pelo território nacional, a compreensão da crise sanitária que se apresentava configurava-se de maneira contraditória, especialmente em decorrência de divergências políticas e científicas entre as instâncias mais elevadas do governo. A consequência são desencontros de condutas e atitudes resultando em insegurança e incerteza na população sobre as melhores condutas a serem adotadas.

Por ampla difusão na imprensa, o presidente da República, Jair Bolsonaro, referia-se a doença como uma simples sindrome gripal, qualificando-a em diversas ocasiões de pronunciamentos oficiais e em redes sociais como uma 'gripezinha', e culpabilizando a mídia por aterrorizar a população. Por outro, o até então ministro da saúde, Luiz Henrique Mandetta, assumiu posição oposta dimensionando a gravidade da pandemia nos parâmetros da OMS e conclamava a população para ficar em casa para evitar a disseminação do vírus e seus impactos deletérios sobre o sistema de saúde.

Somando-se à complexidade das mencionadas rupturas na esfera do governo central e da crise sanitária e política, também se projetava uma crise social e econômica. Neste difícil contexto, teve lugar uma crise ética relacionada ao comportamento de pessoas mal-intencionadas que produziram, publicaram e compartilharam notícias falsas (Fake News) nas redes sociais, aplicativos de mensagem, e até mesmo sites, dificultando a compreensão da magnitude, características e impactos da pandemia(5).

Portanto, esta situação decididamente é complexa, multifacetada e grave, impulsionando os profissionais de saúde a assumirem uma posição de ancoragem em evidências científicas e a buscar fontes confiáveis de difusão da informação.

Canais que atendem critérios de confiabilidade e ampla acessibilidade estão disponiveis para os profissionais de enfermagem, dentre eles, o Portal do Conselho Federal de
Enfermagem (Cofen), que além de servir como importante fonte de consulta para instrumentalizar os profissionais da categoria, dar transparência quanto as ações e deliberações do próprio sistema, de maneira que se passa a compreender aquilo que os gestores têm realizado a partir do poder de representação que thes foi confiado(b)

Por conta das mencionadas características, da problemática apresentada, e sobretudo, em função da atuação dos profissionais de enfermagem implicados neste contexto, mithares deles atuando na linha de frente do combate à pandemia este estudo se justifica, por registrar dados de um evento histórico de proporção mundial - a pandemia da COVID-19. Assim, o presente estudo tem o objetivo de analisar os conteúdos relacionados à COVID-19, veiculados nas publicações do Conselho Federal de Enfermagem (Cofen).

\section{MÉTODO}

\section{Tipo e local de Estudo}

Estudo de abordagem qualitativa, do tipo documental(7). cujas fontes primárias incluíram as reportagens e notícias veiculadas no sítio eletrônico do Cofen (www.cofen.gov.br), especificamente no menu 'Imprensa', página 'notícias'.

\section{Coleta de dados}

A primeira etapa de coleta dos dados consistiu na catalogação das notícias e informativos referentes ao novo coronavírus e a pandemia da COVID-19 em ordem cronológica, publicadas pelo período de 2 meses. O recorte temporal considerou como marco inicial, a data em que à COVID-19 foi determinada como doença de importância internacional (30/01/2020) e como marco final arbitrou-se estabelecer 60 dias após a primeira publicação sobre a temática (14/04/2020).

A segunda etapa da coleta de dados compreendeu a leitura minuciosa do conteúdo das publicações e sua organização por temas. Tal procedimento deu origem a um quadro que permitiu uma visão ampla e, ao mesmo tempo, aprofundada do conteúdo das publicações e dos assuntos divulgados no referido site.

\section{Procedimento de tratamento e análise dos dados}

Os dados provenientes das notícias foram agrupados por semelhança de significados e, à medida que os conteúdos foram analisados, eles eram enquadrados em categorias, permitindo fazer inferências e comparações com outros achados da literatura sobre a temática pesquisada.

\section{Aspectos éticos}

Uma vez que a pesquisa não envolveu diretamente seres 
humanos, e os dados foram coletados em um sítio eletrônico de acesso livre, disponivel a todos que visualizem tal portal e seus conteúdos, não houve necessidade de avaliação do projeto por Comitê de Ética em Pesquisa.

Cabe considerar ainda que, os materiais publicados no site do Cofen que são originários de outras fontes, são devidamente identificados no portal, respeitando-se o direito de autoria dos materiais.

\section{RESULTADOS}

Foram identificadas 113 publicações relacionadas ao novo coronavírus e à pandemia da COVID-19, definindo-se 13 temas, conforme consta na tabela 1 .

Tabela 1 - Distribuição dos temas noticiados e percentual de ocorrência em 113 notícias identificadas do Portal do Cofen. Rio de Janeiro, RJ, Brasil, 2020.

\begin{tabular}{|c|c|c|}
\hline Temas noticiados & $\mathbf{n}$ & $\%$ \\
\hline Materiais de cunho informativo, educativo e científico & 26 & $23,0 \%$ \\
\hline $\begin{array}{l}\text { Ações com foco na proteção social, dos serviços de } \\
\text { saúde e da saúde dos trabalhadores }\end{array}$ & 16 & $14,2 \%$ \\
\hline $\begin{array}{l}\text { Vulnerabilidade, adoecimento e morte de profissionais } \\
\text { da saúde }\end{array}$ & 10 & $8,8 \%$ \\
\hline $\begin{array}{l}\text { Suspensão de atividades e prazos; Prorrogação de datas } \\
\text { e Mudança temporária nos procedimentos de registro de } \\
\text { titulos, inscrições e carteira profissional de identidade }\end{array}$ & 9 & $8,0 \%$ \\
\hline $\begin{array}{l}\text { Homenagens e ações de valorização e reconhecimento da } \\
\text { enfermagem, dos profissionais da saúde e dos sistemas } \\
\text { de saúde público }\end{array}$ & 9 & $8,0 \%$ \\
\hline Avanço da doença e dados epidemiológicos & 7 & $6,2 \%$ \\
\hline Fiscalizações & 7 & $6,2 \%$ \\
\hline Mobilização dos profissionais de enfermagem & 7 & $6,2 \%$ \\
\hline Posicionamento de Entidades frente a atos do governo & 7 & $6,2 \%$ \\
\hline $\begin{array}{l}\text { Reforço da força de trabalho da enfermagem por meio de } \\
\text { contratações emergenciais }\end{array}$ & 6 & $5,3 \%$ \\
\hline $\begin{array}{l}\text { Relatos de experiências de profissionais da saúde durante } \\
\text { a pandemia }\end{array}$ & 4 & $3,5 \%$ \\
\hline Denúncias & 3 & $2,7 \%$ \\
\hline Boas notícias em meio à crise & 2 & $1,8 \%$ \\
\hline Total & 113 & 100 \\
\hline
\end{tabular}

As primeiras divulgações ocorreram em meados do mês de fevereiro (14/02/2020), e até o final do referido mês 03 $(2,7 \%)$ notícias do total de publicações estava disponível. No mês de março, o número de notícias cresceu exponencialmente, adicionando-se mais para 64 (56,6\%) das publicações neste periodo. Nas duas primeiras semanas de abril, contabi- lizou-se mais 46 (40,7\%) notícias relacionadas ao tema.

Das 113 publicações investigadas, predominou matérias de cunho informativo, educativo e científico, com 26 (23,0\%) ocorrências. O primeiro material sobre o coronavírus foi uma nota técnica, noticiada em 14 de fevereiro. Mas, foi no mês de março, principalmente após o dia 11 , que a maior parte dos materiais foram produzidos e socializados.

Figura 1 - Gráfico de crescimento do número de notícias por 30 dias no período de 14 de fevereiro a 14 de abril e respectiva curva de tendência exponencial.

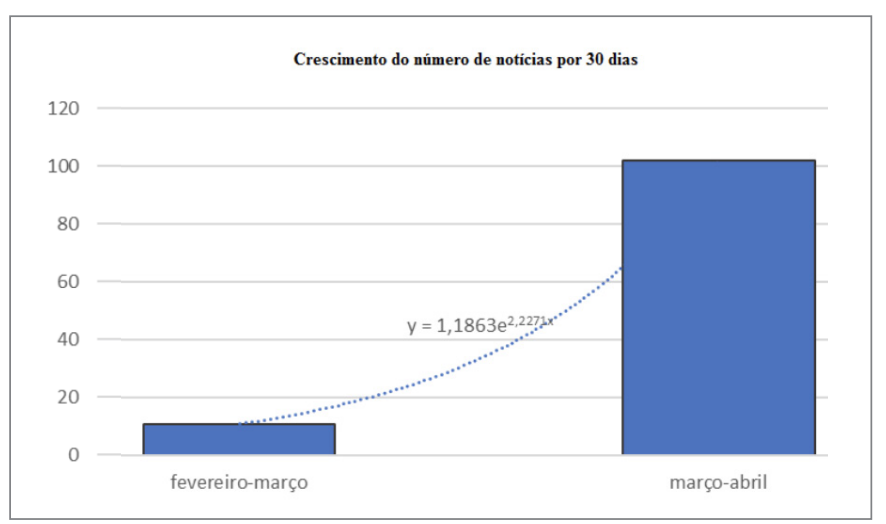

Sete registros apontavam para o avanço da doença em nível mundial e a adoção de estratégias adotadas por instituições como a Fundação Oswaldo Cruz (Fiocruz), para monitorar os dados sobre à COVID-19 no Brasil, e do próprio Cofen, para contabilizar os casos da doença entre os profissionais da enfermagem.

Ações diversas com foco na proteção social, preparação dos serviços de saúde e proteção da saúde física e mental dos trabalhadores, em especial, dos profissionais de enfermagem foram registradas por meio de 16 publicação. $E$, seis outros materiais relatavam o reforço que a força de trabalho em saúde precisaria obter, por meio de contratações emergenciais.

A experiência dos trabalhadores da saúde, em meio à pandemia, foi identificada em 04 publicações. E, a sociedade mostrou-se solidária à causa destes profissionais, passando a fazer recorrentes homenagens, identificadas em 09 produções que salientavam ações de valorização e reconhecimento da enfermagem, dos profissionais da saúde e dos sistemas de saúde público.

Foi possivel ainda notar que, além da atuação direta dos profissionais da saúde nos centros de cuidado aos pacientes, estes profissionais também se mobilizaram e se engajaram em diferentes frentes de atuação. Tal engajamento foi evidenciado em sete publicações as quais divulgaram a 
realização de campanhas tanto em prol da saúde e segurança do coletivo profissional, como também em prol da sociedade, em atos de solidariedade.

E diversas entidades, entre elas os Conselhos de Enfermagem e dos Secretários Estaduais de Saúde, frente à atos do governo, precisaram se posicionar por meio de publicações, em favor da vida, da saúde e da segurança dos profissionais e da sociedade, contrariando declarações e/ ou medidas que vinham sendo divulgadas e defendidas pelo chefe do Executivo. Neste sentido, identificou-se 07 produções, com declarações, manifestações e cartas abertas ao público.

A suspensão de atividades e prazos, além de prorrogações de data e mudança temporária nos procedimentos de registro de títulos, inscrições e carteira profissional de identidade foram documentadas em 09 notícias, divulgadas a partir da segunda quinzena de março

O primeiro registro de fiscalização de unidades de saúde em meio à pandemia foi noticiado em 19 de março e em 14 de abril, os conselhos relataram que já havia fiscalizado 3.772 instituições na pandemia. Sete publicações estavam relacionadas a estas ações de fiscalização e outras três referiam-se, especificamente, a denúncias de falta de equipamentos de proteção individual (EPI) para os profissionais da saúde.

A primeira morte de profissional da enfermagem foi divulgada em 29 de março de 2020. Depois desta, outras notas de pesar registravam perdas de mais profissionais de enfermagem, tanto técnicos de enfermagem quanto enfermeiros, em decorrência da COVID-19. Foi possivel identificar 10 postagens que tratam sobre as vulnerabilidades, adoecimento e morte de trabalhadores da saúde.

Apenas duas postagens apresentavam boas notícias em meio à crise do novo coronavírus, uma tratava sobre a cura de paciente com COVID-19 e a outra, sobre a alta hospitalar de um casal, após vencer a doença.

\section{DISCUSSÃO}

As publicações realizadas pelo Cofen auxiliam os profissionais de saúde, em especial, os da Enfermagem, que ao acessarem os materiais disponíveis conseguem compreender o fenômeno em curso, se estruturar e reestruturar em relação à prática e, assim, prezar pela própria saúde e segurança, como também da população. Salienta-se que tais notícias não se restringiam a discutir temas de interesse da categoria a qual a Entidade defende, sendo em muitas ocasiões de inquietação de todos os profissionais da saúde.

Identificou-se que temas envolvendo a pandemia tornaram-se crescentemente relevantes, gerando inclusive, o aumento de publicações de forma rápida e dinâmica, principalmente após o anúncio da OMS, declarando a doença em escala global. Tal fato pode ser justificado pela necessidade de reavaliação contínua e acelerada dos protocolos para prevenção da COVID-19 entre os trabalhadores expostos ao vírus durante suas atividades laborais ${ }^{(8)}$. Além disso, ao tornar público as ações e decisões tomadas pelos Conselhos, garante-se a transparência, tão importante quanto os atos implementados(b)

O avanço da pandemia, trouxe um impacto enorme à sociedade, alterando hábitos da vida pessoal e relacionados ao trabalho. Além de recomendações gerais para organização dos serviços de saúde e preparo das equipes de enfermagem ${ }^{(9)}$, o Cofen também precisou adequar seus procedimentos de trabalho(10). Por meio da Resolução Cofen 631/2020, autorizou o registro imediato da inscrição profissional após a conclusão de cursos de Enfermagem, na tentativa de suprir rapidamente a carência de profissional nas instituições de saúde ${ }^{(10)}$

Outra medida adotada pelo Cofen foi o cancelamento de eventos ${ }^{(11)}$ num esforço de conter a pandemia da COVID-19 e seguir uma das recomendações não farmacológicas da OMS que é o distanciamento social, visando evitar aglomerações e, principalmente, reduzir a velocidade da transmissão do vírus ${ }^{(12)}$. Ao cancelar eventos, torna-se possivel redirecionar recursos financeiros para a segurança dos profissionais(11)

Uma preocupação latente nas publicações está relacionada ao subdimensionamento das equipes de saúde. Este é um problema antigo, principalmente devido a influência do modelo neoliberal no setor de saúde e à falta de investimento neste âmbito, intensificada a partir da Emenda Constitucional n95, de 15 de dezembro de 2016, que retirou verbas do SUS, congelando investimentos até 2036(13). Neste momento, onde a mão de obra tende a se tornar ainda mais escassa, o Cofen defende que o reforço da força de trabalho da enfermagem deve-se dar por meio de contratações emergenciais ${ }^{(14)}$ e o Conselho Nacional de Saúde (CNS), considerando os prejuízos ao SUS decorrentes da pandemia reivindica revogação imediata da mencionada emenda ${ }^{(13)}$

Por outra via, em meio à crise, os relatos de trabalhadores trouxeram à tona inúmeras situações de sofrimento psicofísico, dentre elas destacam-se: o medo da contaminação, o cansaço físico em decorrência das longas jornadas de trabatho, as lesões de pele em decorrência do uso de EPI, a ansiedade em função das alterações provocadas nos setores de trabalho(15-17). No mundo inteiro, equipes de saúde passaram a trabalhar sob pressão e a rotina dos profissionais para im- 
pedir o avanço da COVID-19 está repleta de estresse, apreensão, incerteza e medo; o que piora o ambiente laboral e a saúde e segurança dos coletivos profissionais ${ }^{(16)}$

$\mathrm{Na}$ China, onde os primeiros casos do vírus foram registrados, um levantamento apontou que cerca de 1,7 mil agentes de saúde foram afetados pela COVID-19, em meio a um sistema de saúde sobrecarregado. E, um dos principais fatores foi a falta de proteção adequada ${ }^{(17)}$.

A falta dos EPI preocupa ${ }^{(18-20)}$ e ganhou destaque nas publicações. $\mathrm{O}$ avanço da pandemia dificultou o abastecimento nacional e internacional destes insumos, assim a OMS vem recomendando seu uso racional ${ }^{(20)}$. Para ajudar neste processo, o Ministério da Saúde definiu, por meio de nota técnica ${ }^{(21)}$, quais EPI devem ser utilizados pelos profissionais, em função das atividades desempenhadas. E o Cofen, lançou cartilha sobre colocação e retirada de EPI ${ }^{(22)}$ e disponibilizou planilha para cálculo e controle destes equipamentos ${ }^{(23)}$.

O Cofen realizou também uma compra emergencial de máscaras do tipo N95 e juntamente com os Conselhos Regionais, tem articulado soluções junto ao poder público para prover os equipamentos necessários aos profissionais, inclusive, Coren de todas as regiões do Brasil têm distribuído materiais visando a proteção dos trabalhadores ${ }^{(24)}$.

Afinal, a falta de proteção para o trabalhador, a nivel individual ou coletivo é uma circunstância que, a partir da interpretação do Código de Ética dos Profissionais de Enfermagem, dá ao trabalhador o direito de recusar-se a exercer atividades ${ }^{(25)}$. E, apesar das fiscalizações dos Conselhos, durante a pandemia, visarem sobretudo contribuir com a estruturação dos serviços de Enfermagem e com o dimensionamento de recursos que serão necessários para fortalecer a capacidade de resposta do sistema de saúde em âmbito nacional, dados consolidados pelo Cofen mostram que os Coren já fiscalizaram 3.772 unidades de saúde por todo o país e, 22.981 profissionais de enfermagem de 1.136 instituições abordadas, denunciaram a falta de máscaras N95/PFF2 para assistência aos pacientes suspeitos ou confirmados da COVID-19(26).

Os Conselhos Regionais de Enfermagem já direcionaram 530 denúncias a órgãos como o Ministério Púbico, Vigilância Sanitária, Secretarias Estaduais e Municipais de Saúde, dentre outros ${ }^{(26)}$. Pois, fornecer EPI e treinar os trabalhadores quanto ao uso são obrigatoriedades das instituições, previstas nas Normas Regulamentadoras do Ministério do Trabalho e Emprego ${ }^{(27-28)}$. Sem EPI e sem treinamento contínuo sobre o seu uso, formas de paramentação e desparamentação, os profissionais da saúde tornam-se alvos fáceis ao adoecimento.
Ademais, não bastasse as vulnerabilidades próprias do exercício profissional, dos riscos inerentes à atividade, ainda há arbitrariedades e medidas de desproteção aos trabalhadores, como é o caso das determinações da Medida Provisória 927, que permite jornadas laborais estendidas e período de descanso diminuído aos profissionais da saúde ${ }^{(29)}$

As determinações da referida medida foram rechaçadas pelo Cofen, que inclusive entrou na justiça pedindo cautela de urgência, para anulá-la, uma vez que, reduz a proteção aos trabalhadores durante a pandemia da COVID-19(29). O Cofen alerta para o adoecimento dos profissionais e aumento de risco de ocorrências diversas por conta da baixa quantitativa de pessoal de enfermagem. Ademais, não se pode esquecer que a crise tem se mostrado uma situação devastadora para subjetividade dos profissionais de enfermagem ${ }^{(30)}$.

A enfermagem tem expressivo quantitativo de profissionais ${ }^{(31)}$, atuando em diversos cenários e expondo-se diariamente a vários riscos ocupacionais, portanto a preocupação com o adoecimento destes profissionais é legítima. Além de resultar em uma baixa significativa nas equipes de trabalho, sobrecarrega aqueles que permanecem na linha de frente ao combate da doença e coloca em risco a população pelo fato de propagação da doença. Faz-se necessário um olhar voltado para a saúde e integridade física e mental dos profissionais de enfermagem, bem como, exige-se que as instituições de saúde proporcionem condições de trabalho para uma atuação segura de acordo com o preconizado no Código de Ética dos Profissionais de Enfermagem.

Infelizmente, até o dia 14 de abril, o novo coronavírus já havia contaminado pelo menos 237 profissionais de enfermagem no país levando 14 a óbito e 12 mortes encontravam-se sob investigação. Outros 2.321 estavam em suspeita de contaminação(32). A vulnerabilidade desses profissionais, o adoecimento e morte, revelam uma situação grave, que exige medidas imediatas, tanto para evitar novas contaminações, quanto para mitigar a exaustão e o adoecimento mental

Diante de tantas dificuldades e temores, também se faz essencial considerar as boas notícias, aquelas que tratam das iniciativas de proteção à saúde do trabalhador e que ressaltam o reconhecimento do importante papel desempenhado pela enfermagem. À medida que a pandemia se estabelecia, esses trabalhadores ganhavam notoriedade e tais publicações trazem acalento aos profissionais que sofrem com a desvalorização da profissão e de seu saber e até então, com a invisibilidade social da categoria ${ }^{(33)}$.

Para salvaguardar a saúde mental dos trabalhadores de enfermagem, o Cofen disponibilizou um canal para aju- 
da emocional a estes profissionais, em meio à situação de pandemia do Coronavírus. O atendimento, conduzido por enfermeiros voluntários, especialistas em saúde mental, é uma ação importante, para ajudar a enfermagem e seus profissionais, a se fortalecer nesse período de crise, buscando supera-la ${ }^{(34)}$

Outra medida que tem efeito positivo sobre a saúde e tem sido observada entre os estes profissionais durante a pandemia, é o engajamento em diferentes frentes, inclusive, envolvendo-se em campanhas solidárias. Além de fortalecer a categoria profissional, essa mobilização compartilhada de cuidado, evoca a sensação de pertença e conforto social(35), renova as esperanças e ajuda a reestabelecer o equilibrio emocional, tão importante neste momento.

\section{Limitação do estudo}

Este estudo apresenta uma limitação temporal, pois as publicações foram coletadas considerando-se apenas o período de dois meses. Ademais, há o fato da crise sanitária ainda está em curso, portanto, até que haja sua normalização, novos dados surgirão e poderão incitar outras discussões.

\section{Contribuições do estudo}

A pesquisa documental online procurou superar o desafio relacionado à execução de uma pesquisa em pleno decorrer de uma crise de saúde. Assim, o uso da tecnologia e o acesso aos materiais publicados pelo Cofen mostraram-se como importantes aliados, à medida que serviram de base para a compreensão da pandemia e o seu avanço, além dos impactos desta doença nos serviços de saúde e nos trabalhadores de enfermagem.

Sugere-se o aprofundamento e a ampliação de estudos sobre a experiência dos trabalhadores de enfermagem envolvidos no combate à pandemia da COVID-19 em momento pós-crise bem como a realização de outras pesquisas documentais que possam ajudar a contar a história da pandemia e a atuação dos profissionais de enfermagem que atuaram na linha de frente desta batalha.

\section{CONCLUSÕES}

Conclui-se que as notícias cresceram a medida que a disseminação da COVID-19 tornou-se uma pandemia. Os materiais de cunho informativo, educativos e científico predominaram entre os temas noticiados, as denúncias de condições de trabalho inadequadas foram avultando-se ao longo dos 2 meses. O posicionamento do Cofen frente a atos do governo bem como as ações preventivas e fiscalizadoras desenvolvidas pelo sistema Cofen/Coren mostram a atuação destas entidades que zelam pelo cumprimento legal da profissão, especialmente em um momento de tanta vulnerabilidade dos profissionais de enfermagem.

Ressalta-se que, quanto ao conteúdo das publicações referentes às boas notícias, este precisa ser explorado e ampliado. No entanto, cabe considerar que as publicações apresentavam principalmente caráter informativo, apesar de trazer alertas preocupantes. Assevera-se que o conteúdo dos materiais socializados não tinha o objetivo de instaurar o medo, mas que os trabalhadores pudessem ter ciência dos acontecimentos que atingiam a categoria e para que pudessem se proteger e se mobilizar em busca de condições de trabalho.

Por meio deste estudo, verificou-se os impactos que a pandemia tem causado. Ademais, constatou-se que o material publicado auxilia os profissionais a compreenderem vários aspectos da COVID-19 e suas implicações para os trabalhadores, instrumentaliza-os com conteúdo de qualidade, garantindo-lhes fundamentos para uma prática segura.

\section{CONTRIBUIÇÕES DOS AUTORES}

Soares SSS, Souza NVDO, Silva KG - concepção e desenho do estudo; coleta, análise e interpretação dos dados; redação e revisão crítica do manuscrito; aprovação da versão final a ser publicada; Costa CCP, Brandão APCL, Carvalho CS, Lacerda AC, Aperibense PGGS - análise e interpretação dos dados; redação e revisão crítica do manuscrito

\section{REFERENCIAS}

1. World Health Organization. Coronavirus disease 2019 (COVID-19) Situation Report - 90. 2020 [cited 2020 Apr 19]. Available from: https://www.who.int/docs/default-source/coronaviruse/situation-reports/20200419-sitrep-90-COVID-19.pdf?sfvrsn=55ld47fd_4. Accessed: 19 Apr 2020

2. World Health Organization (WHO). International Health Regulations (2005). 3a Edição (2016). Available from: <https:// www.who.int/ihr/publications/9789241580496/en/> [Acesso em 15 Apr 2020].

3. Conselho Federal de Enfermagem (Brasil). Enfermagem tem papel fundamental no combate ao coronavirus. [Internet]. 2020 [cited 2020 Apr 16]. Available from: http://www.cofen.gov.br/enfermagem-tem-papel-fundamental-no-combate-ao-coronavirus_77187.html 
4. BBC Brasil. 'Em colapso': a dramática situação dos hospitais da Itália na crise do coronavirus. 2020 Mar 19 [cited 2020 Apr 08]. Available from: https://www.bbc.com/portuguese/internacional-51968491

5. Neto M, Gomes T de O, Porto FR, Rafael R de MR, Foseca MHS, Nascimento J. Fake news no cenário da pandemia da COVID-19. Cogitare enferm. [Internet]. 2020 [cited 20 Apr 2020]; Available from: http://dx.doi.org/10.5380/ ce.v25i0.72627.

6. Lima LMN, Andrade SR, Ruoff AB, Albuquerque GL. Decisões dos Conselhos de Enfermagem no Brasil: uma pesquisa documental. Enferm. Foco 2017; 8 (4): 42-48. Doi: https://doi.org/10.21675/2357-707X.2017.v8.n4.1328

7. Cellard André. A análise documental. In: Poupart J, Deslauriers JP, Groulx LH, Laperriére A, Mayer R, Pires A. A pesquisa qualitativa: enfoques epistemológicos e metodológicos. Petrópolis: Vozes; 2008. p.295-316.

8. Gallasch CH, Cunha ML, Pereira LAS, Silva Junior, JS Prevenção relacionada à exposição ocupacional do profissional de saúde no cenário da COVID-19. Rev enferm UERJ, Rio de Janeiro, 2020; 28:e 49596 DOI: http://dx.doi. org/10.12957/reuerj.2020.49596

9. Conselho Federal de Enfermagem (Brasil). Recomendações Gerais para Organização dos Serviços de Saúde e Preparo das Equipes de Enfermagem. [Internet] 2020 [cited 2020 Apr 26]. Available from: http://www.cofen.gov.br/ wp-content/uploads/2020/03/cofen_COVID19_comp.pdf

10. Conselho Federal de Enfermagem (Brasil). Resolução Cofen n으 631 de 23 de março de 2020. Altera, em caráter excepcional, "ad referendum" do Plenário do Cofen, em virtude da situação gerada pela pandemia da COVID-19, os processos administrativos de atendimento ao profissional referentes ao registro de títulos, concessão de inscrição, inscrição remida, suspensão de inscrição, cancelamento e reinscrição, inscrição secundária, substituição e renovação da carteira profissional de identidade e transferência de inscrição, e dá outras providências. Brasilia (DF). Diário Oificial da União. 23 mar 2020.

11. Conselho Federal de Enfermagem (Brasil). Cofen suspende 23을 Congresso Brasileiro dos Conselhos de Enfermagem. [Internet]. 2020 [cited 2020 Apr 27]. Available from: http://www.cofen.gov.br/cofen-suspende-23o-congresso-brasileiro-dos-conselhos-de-enfermagem_78326.html

12. Yuzhen Z, Jiang B, Yuan J, Tao Y. The impact of social distancing and epicenter lockdown on the COVID-19 epidemic in mainland China: a data-driven SEIQR model study. Med Rxiv[Internet]. 2020 [cited 2020 Apr 26]. DOI: https://doi.org/10.1101/2020.03.04.20031187.

13. Conselho Nacional de Saúde (Brasil). Nota pública CNS reinvindica revogação imediata da EC 95/2016 para enfrentamento ao coronavirus. 2020 Mar 14 [cited 2020 Apr 25]. In: Conselho Federal de Enfermagem [Internet]. CNS quer revogar emenda 95/2016, que prejudica reação ao coronavirus. Available from: http://www.cofen. gov.br/cns-reivindica-revogacao-imediata-de-emenda-que-retirou-verba-do-sus-prejudicando-enfrentamento-ao-coronavirus_77846.html

14. Conselho Federal de Enfermagem (Brasil). Conselhos de Enfermagem pedem contratação emergencial de profissionais. [Internet]. 2020 [cited 2020 Apr 27]. Available from:http://www. cofen.gov.br/conselhos-de-enfermagem-pedem-contratacao-emergencial-de-profissionais_77981.html

15. O Estado de São Paulo. Enfermeira comove internautas com relato sobre trabalho em meio ao coronavirus. 2020 Mar 11 [cited 2020 Apr 20]. In: Conselho Federal de Enfermagem [Internet]. Enfermeira comove internautas com relato sobre trabalho em meio ao coronavírus. 2020 Mar 12. Available from: http://www.cofen.gov.br/enfermeira-comove-internautas-com-relato-sobre-trabalho-em-meio-ao-coronavirus_77790.html

16. Record TV. Médicos e enfermeiros revelam como é a rotina no combate ao coronavirus. 2020 Apr 06 [cited 2020 Apr 17]. In: Conselho Federal de Enfermagem [Internet]. Available from: http://www.cofen.gov.br/medicos-e-enfermeiros-revelam-como-e-a-rotina-no-combate-ao-coronavirus_78706.html

17. Lemos V. BBC Brasil. Achei que tinha sido infectada ao atender paciente': a rotina de profissionais de saúde que cuidam de casos de coronavirus no Brasil. 2020 Mar 16 [cited 2020 Apr 16]. In: Conselho Federal de Enfermagem [Internet]. Available from: http://www.cofen.gov.br/ achei-que-tinha-sido-infectada-a-rotina-de-profissionais-que-cuidam-de-casos-de-coronavirus_77862.html

18. Darlenski R,Tsankov N. COVID-19 pandemic and skin - What should dermatologists know? Doi: 10.1016/j.clindermatol.2020.03.012

19. Conselho Federal de Enfermagem (Brasil). Cancelamento de compra de EPI preocupa o Cofen. [Internet] 2020 [cited 2020 Apr 27]. Available from: http://www. cofen.gov.br/cancelamento-de-compra-de-epis-preocupa-o-cofen_78598.html 
20. World Health Organization. Rational use of personal protective equipment (PPE) for coronavirus disease (COVID-19). 2020 [cited 2020 Apr 08]. Available from: https://apps.who.int/iris/bitstream/ handle/10665/331498/WHO-2019-nCoV-IPCPPE_use2020.2-eng.pdf

21. Brasil. Agência Nacional de Vigilância Sanitária. Nota Técnica 04/2020. Orientações para serviços de saúde: medidas de prevenção e controle que devem ser adotadas durante a assistência aos casos suspeitos ou confirmados de infecção pelo novo coronavírus (SARS-CoV-2). [Internet]. 2020 [cited 2020 Apr 25]. Available from: http://portal.anvisa.gov.br/documents/33852/271858/Nota+T\%C3\%A9cni$c a+n+04-2020+G V I M S-G G T E S-A N V I S A / a b 598660-3 d e 4-$ 4f14-8e6f-b934lc196b28

22. Conselho Federal de Enfermagem (Brasil). Orientações sobre a colocação e retirada dos equipamentos de proteção individual (EPIs). [Internet] 2020 [cited $2020 \mathrm{Apr}$ 28]. Available from: http://www.cofen.gov.br/wp-content/ uploads/2020/03/cartilha_epi.pdf

23. Conselho Federal de Enfermagem (Brasil). Cofen disponibiliza planilha de cálculo e controle de EPIs. [Internet]. 2020 [cited 2020 Apr 29]. Available from: http:// www.cofen.gov.br/cofen-disponibiliza-planilha-de-calculo-e-controle-de-epis_78574.html

24. Conselho Federal de Enfermagem (Brasil). Cofen lança edital para compra de máscaras N95. [Internet]. 2020 [cited 2020 Apr 27]. Available from: http://www.cofen. gov.br/cofen-lanca-edital-para-compra-de-mascaras-n95-2_78292.html

25. Conselho Federal de Enfermagem (Brasil). Resolução Cofen no 564 de 6 de novembro de 2017. Aprova o novo Código de Ética dos Profissionais de Enfermagem. Brasilia (DF). Diário Oificial da União. 06 nov 2017.

26. Conselho Federal de Enfermagem (Brasil). Conselhos de Enfermagem fiscalizaram 3.772 instituições na pandemia. [Internet]. 2020 [cited 2020 Apr 17]. Available from: http://www.cofen.gov.br/conselhos-de-enfermagem-fiscalizaram-3-772-instituicoes-na-pandemia_79098.html

27. Ministério do Trabalho e Emprego (BR). Norma Regulamentadora 6 - Equipamento de Proteção Individual - EPI. [Internet]. [cited 2020 Apr 18]. Available from: https://enit. trabalho.gov.br/portal/images/Arquivos_SST/SST_NR/ NR-06.pdf

28. Ministério do Trabalho e Emprego (BR). Portaria n으
485, de 11 de novembro de 2005. Aprova a norma regulamentadora no 32 (Segurança e saúde no trabalho em estabelecimentos de saúde) [Internet]. Diário Oficial da República Federativa do Brasil, Brasilia (DF); 2005 [cited 2020 Apr 10]. Available from: http://sbbq.iq.usp.br/arquivos/seguranca/portaria485.pdf

29. Conselho Federal de Enfermagem (Brasil). Cofen vai à justiça contra a Medida Provisória 927. [Internet]. 2020 [cited 2020 Apr 27]. Available from: http://www.cofen.gov.br/ cofen-vai-a-justica-contra-medida-provisoria-927_78489. html

30. Puente-Fernández D, Lozano-Romero MM, Montoya-Juárez R, Marti-García C, Campos-Calderón C, Hueso-Montoro C. Nursing professionals' attitudes, strategies, and care practices towards death: a systematic review of qualitative studies. J Nurs Scholarsh. 2020; doi: https://doi. org/10.11ll/jnu.12550

31. Machado MH, Filho WA, Lacerda WF, Oliveira E, Lemos $W$, Wermelinger $M$, et al. Caracteristicas gerais da enfermagem: o perfil sócio demográfico. Enferm. Foco [Internet]. 2016 [citted Apr 27];6 (4):11-17. Available from: http:// revista.portalcofen.gov.br/index. php/enfermagem/article/ view/686/296

32. CNN Brasil. Coronavírus já contaminou 237 profissionais de enfermagem no país. 2020 Mar 13[cited $2020 \mathrm{Apr}$ 19]. In: Conselho Federal de Enfermagem [Internet]. 2020 Apr 14. Available from: http://www.cofen.gov.br/coronavirus-ja-contaminou-237-profissionais-de-enfermagem-no-pais_79067.html

33. Conselho Regional de Enfermagem (Paraná). Demandas de décadas da Enfermagem se sobressaem no combate à pandemia. 2020 Apr 09 [cited 2020 Apr 27]. In: Conselho Federal de Enfermagem [Internet]. Available from: http:// www.cofen.gov.br/demandas-de-decadas-da-enfermagem-se-sobressaem-no-combate-a-pandemia_78927.html

34. Conselho Federal de Enfermagem (Brasil). Cofen disponibiliza canal para ajuda emocional a profissionais [Internet]. 2020 [cited 2020 Apr 27]. Available from: http://www. cofen.gov.br/cofen-disponibiliza-canal-para-ajuda-emocional-a-profissionais_78283.html

35. Ministério da Saúde (BR). Fundação Oswaldo Cruz - Fiocruz - Saúde mental e atenção psicossocial na pandemia COVID-19 cartilha recomendações gerais. [Internet] 2020 [cited 2020 Apr 26]. Available from: http://renastonline.ensp.fiocruz.br/sites/default/ files/arquivos/recursos/cartilha_recomendacoes_gerais_06_ 04.pdf.pdf 